PAPER

Atmospheric adsorption on pristine and nitrogen-doped graphene: doping-dependent, spatially selective

To cite this article: Egor A Kolesov et al 2020 J. Phys. D: Appl. Phys. 53045302

View the article online for updates and enhancements.

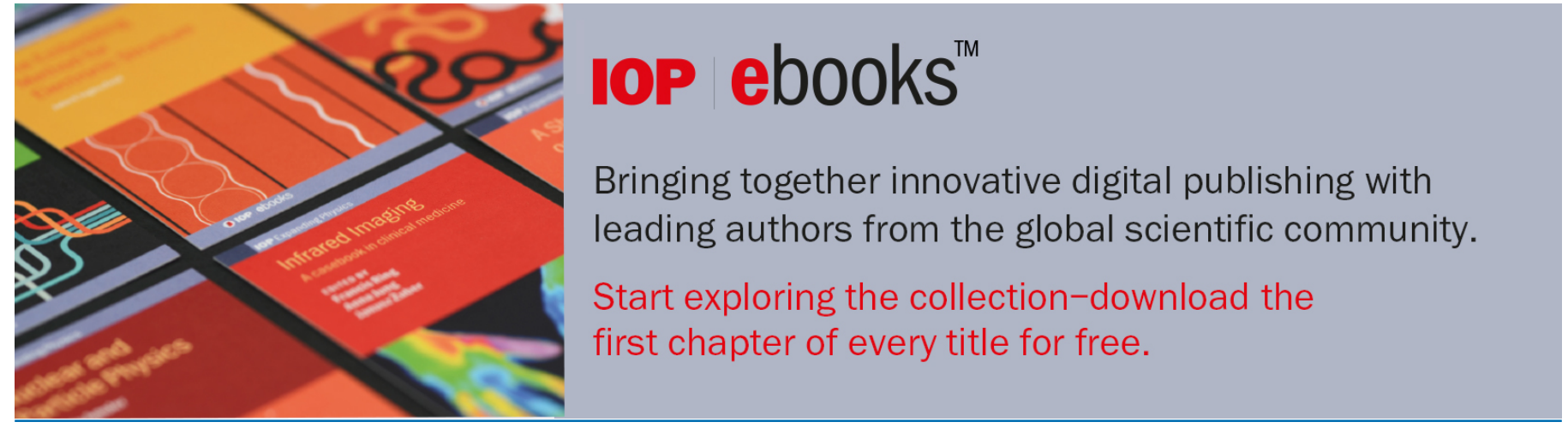

This content was downloaded from IP address 131.188 .6 .12 on $09 / 06 / 2020$ at $18: 38$ 


\title{
Atmospheric adsorption on pristine and nitrogen-doped graphene: doping-dependent, spatially selective
}

\author{
Egor A Kolesov ${ }^{1}$, Mikhail S Tivanov ${ }^{1,7,8}$, Olga V Korolik ${ }^{1}$, \\ Elmar Yu Kataev' ${ }^{2}$ Fu Xiao ${ }^{3}$, Olesya O Kapitanova ${ }^{4,5}$, Hak Dong Cho ${ }^{3}$, \\ Tae Won Kang ${ }^{3}$ and Gennady N Panin ${ }^{3,6}$ (0) \\ 1 Faculty of Physics, Belarusian State University, 4 Nezavisimosti Av., 220030 Minsk, Belarus \\ 2 Chair of Physical Chemistry II, Friedrich-Alexander-University of Erlangen-Nürnberg, Egerlandstraße, \\ 3, 91052, Erlangen, Germany \\ ${ }^{3}$ Department of Physics, Quantum-Functional Semiconductor Research Center, Nano Information \\ Technology Academy, Dongguk University, 3-26 Pildong, Junggu, 100-715, Seoul, Republic of Korea \\ ${ }^{4}$ Department of Chemistry, Moscow State University, Leninskie Gory, 1, b.3, 119991, Moscow, Russia \\ 5 Moscow Institute of Physics and Technology, 9 Institutskiy per., Dolgoprudny, Moscow Region, \\ 141701, Russia \\ ${ }^{6}$ Institute for Microelectronics Technology and High Purity Materials, RAS, 142432 Chernogolovka, \\ Moscow district, Russia
}

E-mail tivanov@bsu.by

Received 6 September 2019, revised 22 October 2019

Accepted for publication 30 October 2019

Published 18 November 2019

\begin{abstract}
Using Raman and photoemission spectroscopy, we probe the atmospheric adsorption on pristine and nitrogen $p$-doped graphene supported by $\mathrm{SiO}_{2} / \mathrm{Si}$. Laser annealing in vacuum led to a pronounced change in Raman spectra parameters, corresponding to a decrease in hole density due to adsorbate removal from the sample surface. We found that the shift inversely correlates with a degree of initial nitrogen doping, and thus less $p$-type adsorption doping takes place on graphene with a higher density of charge carriers with the same sign. The amount of hole doping required for the absence of atmospheric adsorption doping was found to be $p_{\text {noad }}=(3.87 \pm 0.31) \times 10^{13} \mathrm{~cm}^{-2}(\sim 2.3 \%$ of pyridine-like nitrogen $)$, while the electronic doping required to fully compensate adsorption hole doping was $n_{\text {equil }}=(0.46 \pm 0.12) \times 10^{13} \mathrm{~cm}^{-2}(\sim 0.2 \%$ of graphitic nitrogen $)$. We showed that atmospheric adsorption on pristine graphene increases the spatial charge carrier inhomogeneity; in contrast, the adsorption on nitrogen-doped graphene shows a spatially selective nature and reduces the inhomogeneity of charge carriers. This study is useful for graphene applications which require specific adsorption properties, as well as for taking these properties into account when designing graphene-based nanoelectronic devices.
\end{abstract}

Keywords:graphene, adsorption, doping, Raman spectroscopy, x-ray photoelectron spectroscopy

S Supplementary material for this article is available online

(Some figures may appear in colour only in the online journal)

\footnotetext{
${ }^{7}$ Author to whom any correspondence should be addressed.

84 Nezavisimosti Av., 220030 Minsk, Belarus
} 


\section{Introduction}

Graphene, a monolayer $\mathrm{sp}^{2}$ carbon allotrope, has become popular due to its extremely promising physical properties [1]. Among them, one can highlight the strong sensitivity of this 2D material to adsorbates [2-9]. On one hand, this leads to graphene being widely studied and engineered towards sensor applications [2]; on the other, such sensitivity causes significant modification of graphene properties, even when the material is simply air-exposed [3-8]. In particular, atmospheric adsorption leads to integral $p$-type doping in graphene due to acceptor $\mathrm{H}_{2} \mathrm{O}$ and $\mathrm{O}_{2}$ adsorbates [3-8]. This effect can be undesirable for graphene-based nanoelectronic devices operating in air; at the same time, such atmospheric adsorption phenomena make graphene a perspective material for chemical and environmental sensors, selective catalysis and other applications [2].

Since the atmospheric adsorption leads to a charge carrier density change in graphene, the preliminarily doped material can demonstrate different adsorption behavior compared to the pristine one $[8,10]$. Recently we showed [8] that $n$-type doping of graphene leads to stronger atmospheric adsorption; furthermore, the adsorption tends to counterbalance both the initial 'excess' electronic density and spatial charge carrier inhomogeneity induced by the dopants. Since fully or partially opposite can be logically expected for the $p$-type doping case, this effect is interesting in terms of a range of possible applications. Thus, investigating the relationship between initial and atmospheric doping, as well as comparing the behavior of pristine and doped graphene, can open up new prospects for controlling the adsorption properties of this material, as well as extend the understanding of the effect.

A natural way to probe atmospheric adsorption on a $2 \mathrm{D}$ material is to conduct experiments under an air environment and in vacuum [8]; additionally, annealing is widely used to remove the adsorbates from the sample surface.

Raman spectroscopy is a versatile and non-destructive classical tool for obtaining information about the properties of graphene [11]. Several different Raman features of this material are known to be non-linearly doping-dependent [12], and thus they make it possible to determine the charge carrier sign or charge carrier density variations, as well as distinguish these from the substrateinduced strain in terms of the effect on the Raman spectra [13, 14]. $\mathrm{X}$-ray photoelectron spectroscopy (XPS) is another classical quantitative tool that allows one to determine both the elemental composition and atomic chemical bonding type [15]. Combining these complementary methods allows one to obtain information on how adsorption on graphene occurs, depending on the presence and amount of initial doping of the material.

The purpose of this study is to probe and compare atmospheric adsorption on pristine and $p$-doped graphene in terms of the effect on charge carrier density by Raman and photoemission spectroscopy at normal pressure and upon laser annealing in vacuum.

\section{Experimental}

Within the scope of this work, we define atmospheric adsorption as a self-sustained adsorption of ambient air components, among which $\mathrm{H}_{2} \mathrm{O}$ and $\mathrm{O}_{2}$ adsorbates act as dopants, on the experimental graphene [3-8].

Graphene was synthesized by chemical vapor deposition (CVD) at $1020{ }^{\circ} \mathrm{C}$ on $\mathrm{Cu}$ with $\mathrm{CH}_{4}$ flow of $40 \mathrm{sccm}$ and hydrogen flow of $10 \mathrm{sccm}$. The copper foil substrate, $99.999 \%$ pure Alfa Aesar, with a thickness of $25 \mu \mathrm{m}$ and area of $10 \times 30 \mathrm{~cm}^{2}$, was pre-annealed for $1-2 \mathrm{~h}$ inside the synthesis chamber at $1060{ }^{\circ} \mathrm{C}$ under the flow of hydrogen $(300 \mathrm{sccm})$ and argon $(2000 \mathrm{sccm})$, with a base pressure less than $10^{-4}$ Torr. After the growth of graphene, the substrate was cooled down to room temperature at a rate described in detail elsewhere [16].

Graphene transfer to $\mathrm{SiO}_{2} / \mathrm{Si}$ was performed using a 'PMMA-mediated' method [17]. PMMA (Polymethyl methacrylate) with a molecular weight of $996000 \mathrm{~g} / \mathrm{mol}$, dissolved in anisole, was spin-coated (3000 rpm, $1 \mathrm{~min}$ ) on graphene supported by copper foil. Then, an aqueous solution of $0.1 \mathrm{M}$ $\left(\mathrm{NH}_{4}\right)_{2} \mathrm{~S}_{2} \mathrm{O}_{8}$ was utilized for copper etching; the etching products were removed from graphene using a water/isopropyl alcohol mixture [18]. To remove the PMMA, the sample was kept in extra-pure glacial acetic acid [19] for $4 \mathrm{~h}$.

Graphene was doped with atomic nitrogen generated by a magnetron discharge (ParaOne, PS-1200) with $5 \mathrm{sscm} \mathrm{N}_{2}$ flow in a vacuum chamber, with the base pressure being less than 3 . $10^{-4}$ mbar. The plasma power was $10 \mathrm{~W}$, while the processing time was 30 s (sample N1), 90s (N2) or 60s (N3). Such labelling is related to the resultant nitrogen concentration rather than the treatment time, and is explained in detail later in the results and discussion section. Changing the plasma power to $20 \mathrm{~W}$ did not noticeably affect the results.

To obtain Raman spectra, a Nanofinder HE (LOTIS TII) confocal Raman spectrometer was used. The spectral resolution was better than $3 \mathrm{~cm}^{-1}$ (about $2.3 \mathrm{~cm}^{-1}$ for the $\mathrm{G}$ peak and about $1.0 \mathrm{~cm}^{-1}$ for the $2 \mathrm{D}$ peak). Raman radiation was excited with a $532 \mathrm{~nm}$ continuous solid-state laser. The spectra measurements were carried out in a pressure-controlled cell under an atmospheric environment or vacuum (less than $5 \times 10^{-4}$ $\mathrm{Pa}$ ) using laser power of $3 \mathrm{~mW}$ for $30 \mathrm{~s}$ exposition per spectrum; the laser spot diameter was $\sim 1.5 \mu \mathrm{m}$.

Due to the potential to perform area mapping and change the laser power, a Raman source laser was used within the scope of this work, both to obtain spatial spectra scans and as a heat source for the same area point-by-point consistent annealing. The $10 \mathrm{~mW}$ power was empirically chosen for the annealing as a maximal value that does not affect graphene defectiveness in terms of $\mathrm{D}$ and $\mathrm{D}^{\prime}$ peak relative intensity [11]. The $3 \mathrm{~mW}$ power was proven not to affect the spectra through repeated measurements at the same point. Prior to the adsorption-related measurements, the samples were exposed to ambient air with a relative humidity ranging from $40 \%$ to $60 \%$ for at least two months. Then, Raman scans of $6 \times$ $6 \mu \mathrm{m}^{2}$ (49 points per scan) were performed for the same areas of the experimental samples within the following sequence: measurement (3 mW, air-exposed)_annealing (10 $\mathrm{mW}$, vacuum)—measurement ( $3 \mathrm{~mW}$, vacuum). The measurements were performed under room temperature ambience.

Photoemission spectra were acquired using a PHI Quantera II spectrometer equipped with a monochromatized $\mathrm{Al} \mathrm{K} \alpha$ 


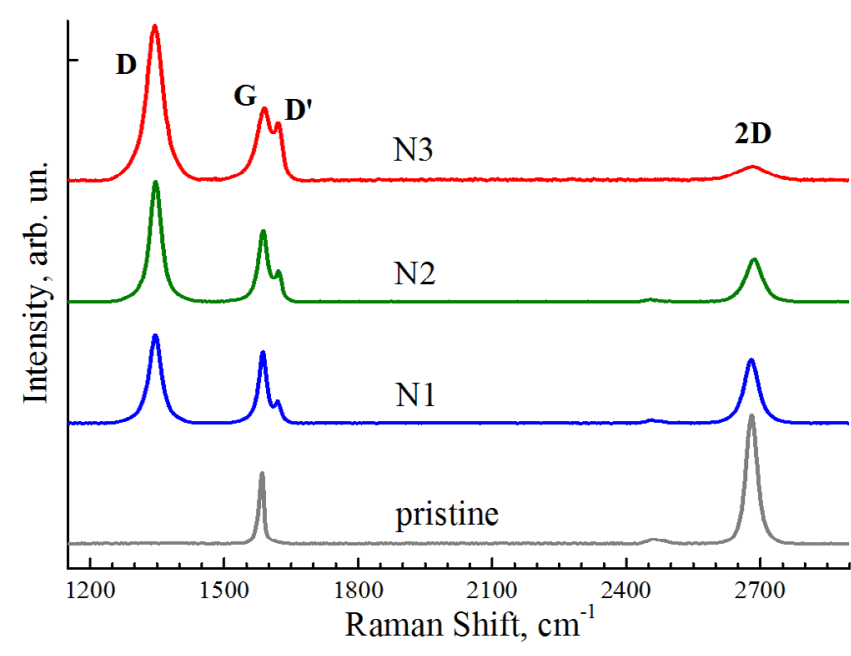

Figure 1. Typical Raman spectra of pristine and nitrogen-doped graphene N1-N3 under an atmospheric environment.

X-ray source (1486.6eV energy) and a combined ion/electron charge neutralization gun. The spectra were fitted by Gaussian/Lorentzian convolution functions using a Unifit 2014 data processor. Asymmetry of the $\mathrm{sp}^{2}$ component in $\mathrm{C} 1 \mathrm{~s}$ core level spectra was described with Doniach-Sŭnjić functions. The spectral background was optimized using a combination of Shirley and Tougaard functions simultaneously with the spectral fitting. Atomic fractions were calculated from peak areas using calibrated sensitivity factors.

\section{Results and discussion}

We first investigate the structure and composition of pristine and doped graphene samples under atmospheric conditions. Secondly, we focus on the nature and type of doping in the nitrogen-treated graphene samples. Finally, we discuss the change in carrier density and structural features of pristine and doped graphene samples upon laser annealing in vacuum.

Raman spectra of pristine and nitrogen-doped graphene are shown in figure 1 . For all samples, the $\mathrm{G}$ and $2 \mathrm{D}$ peaks characteristic of graphene are present [11]. The spectra of N1-N3 samples treated with nitrogen plasma are typical of doped graphene: they show pronounced $\mathrm{D}$ and $\mathrm{D}^{\prime}$ peaks which arise in the presence of defects [11]. Furthermore, a smaller relative intensity is observed for the 2D peak, which is known to be suppressed by disorder and additional charge carriers [11, 12]. Alongside both $\mathrm{G}$ and $2 \mathrm{D}$ peak upshifts of $3-6 \mathrm{~cm}^{-1}$, generally observed in the spectra of nitrogen-treated experimental samples, these features are indicative of a $p$-type doping [12]. The upshift increases from sample N1 to N3, the D and $\mathrm{D}^{\prime}$ peaks become more pronounced, while the $2 \mathrm{D}$ peak relative intensity decreases, all indicating a doping increase [12].

The surface composition measured by XPS clearly indicates the incorporation of nitrogen in the graphene structure. In figure 2, we present the $\mathrm{C} 1 \mathrm{~s}$ and $\mathrm{N} 1 \mathrm{~s}$ photoemission spectra for N1-N3 samples, taken after $\mathrm{N}_{2}$ plasma treatment (the survey spectra are given in the supplementary material file 1, figure S1 (stacks.iop.org/JPhysD/53/045302/mmedia)). A summary of the atomic concentrations is presented in table 1 .
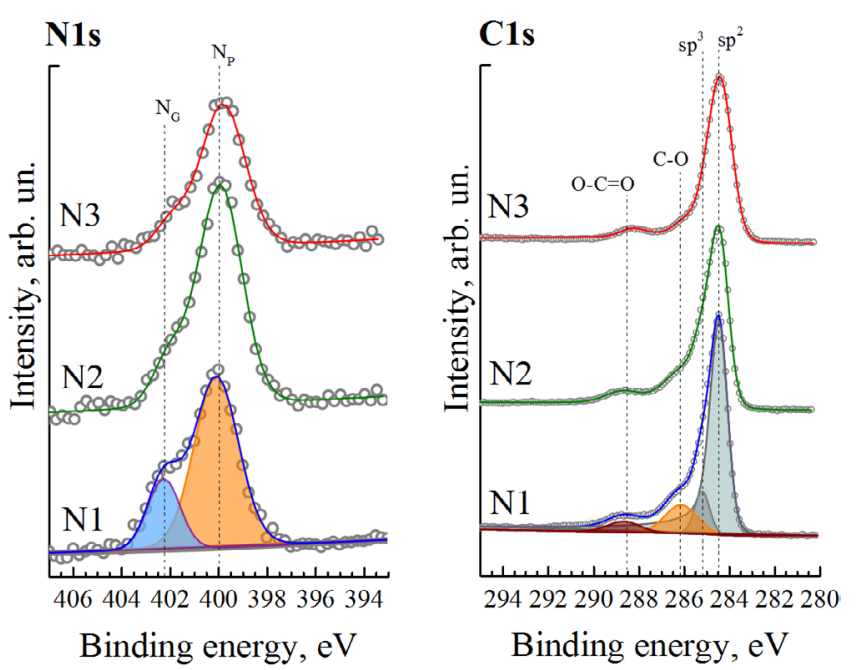

Figure 2. N1s (a) and C1s (b) photoemission spectra acquired after the $\mathrm{N}_{2}$ plasma treatment for $30 \mathrm{~s}(\mathrm{~N} 1), 90 \mathrm{~s}(\mathrm{~N} 2)$ and $60 \mathrm{~s}(\mathrm{~N} 3)$. The labels $\mathrm{N}_{\mathrm{P}}$ and $\mathrm{N}_{\mathrm{G}}$ correspond to pyridinic and graphitic nitrogen, respectively.

Table 1. Atomic concentrations of carbon, $\mathrm{sp}^{2}$ carbon, pyridinic and graphitic nitrogen in the graphene samples, treated with $\mathrm{N}_{2}$ plasma and transferred to $\mathrm{SiO}_{2} / \mathrm{Si}$ substrate.

\begin{tabular}{lllllll}
\hline Sample & $\mathrm{C}, \%$ & $\mathrm{C}_{\mathrm{sp} 2}, \%$ & $\begin{array}{c}\mathrm{C} / \mathrm{Si} \\
\text { ratio }\end{array}$ & $\mathrm{N}_{\mathrm{P}}, \%$ & $\mathrm{~N}_{\mathrm{G}}, \%$ & $\begin{array}{c}\mathrm{C}_{\mathrm{sp}}^{2} / \mathrm{N} \\
\text { ratio }\end{array}$ \\
\hline $\mathrm{N} 1$ & 97.8 & 72.7 & 2.61 & 1.7 & 0.5 & 27.5 \\
$\mathrm{~N} 2$ & 97.5 & 88.4 & 1.40 & 2.2 & 0.3 & 25.4 \\
$\mathrm{~N} 3$ & 97.3 & 71.0 & 2.56 & 2.3 & 0.4 & 21.2 \\
\hline
\end{tabular}

We describe the $\mathrm{C} 1 \mathrm{~s}$ spectrum by the intense component at $284.5 \mathrm{eV}$ binding energy corresponding to $\mathrm{sp}^{2} \mathrm{C}-\mathrm{C}$ bonding in the graphene lattice $[20,21]$. Other spectral components are related to different carbon species in PMMA residuals according to [22]: $\mathrm{C}-\mathrm{C} \mathrm{sp}{ }^{3}$ at $285.2 \mathrm{eV}, \mathrm{C}-\mathrm{O}$ at $286.2 \mathrm{eV}$ and $\mathrm{O}-\mathrm{C}=\mathrm{O}$ at $288.5 \mathrm{eV}$. The $\mathrm{N} 1 \mathrm{~s}$ spectra can be described by two components labeled $\mathrm{N}_{\mathrm{P}}$ and $\mathrm{N}_{\mathrm{G}}$ at 400.0 and $402.2 \mathrm{eV}$, respectively, associated with pyridinic and graphitic nitrogen [23, 24]. The peak at $400.0 \mathrm{eV}$ can also relate to amine and pyrrolic impurities; however, these incorporated nitrogen configurations are more likely to form under $\mathrm{NH}_{3}$ treatment [25] rather than $\mathrm{N}_{2}$, used in the present work, which is known to mainly introduce pyridinic and graphitic sites [26]. Nevertheless, we do not exclude the possibility of the presence of small quantities of pyrrolic and amine nitrogen, undistinguishable in N1s spectra.

For the bombardment time of $90 \mathrm{~s}$ (N2), we observe a following decrease in $\mathrm{N}$ functionalities compared to the $60 \mathrm{~s}$ treatment (N3). This change in the N1s spectra is in consensus with the $\mathrm{C} / \mathrm{Si}$ intensity ratio, confirming the partial carbon atom removal from graphene on $\mathrm{SiO}_{2} / \mathrm{Si}$ substrate under 90 s plasma treatment. It indicates that at this bombardment time, the doping procedure becomes less productive, and the defects introduced by nitrogen plasma treatment are no longer associated with dopants; instead, nitrogen atoms already incorporated in the graphene lattice are subsequently removed. While the Raman spectra are consistent with these 

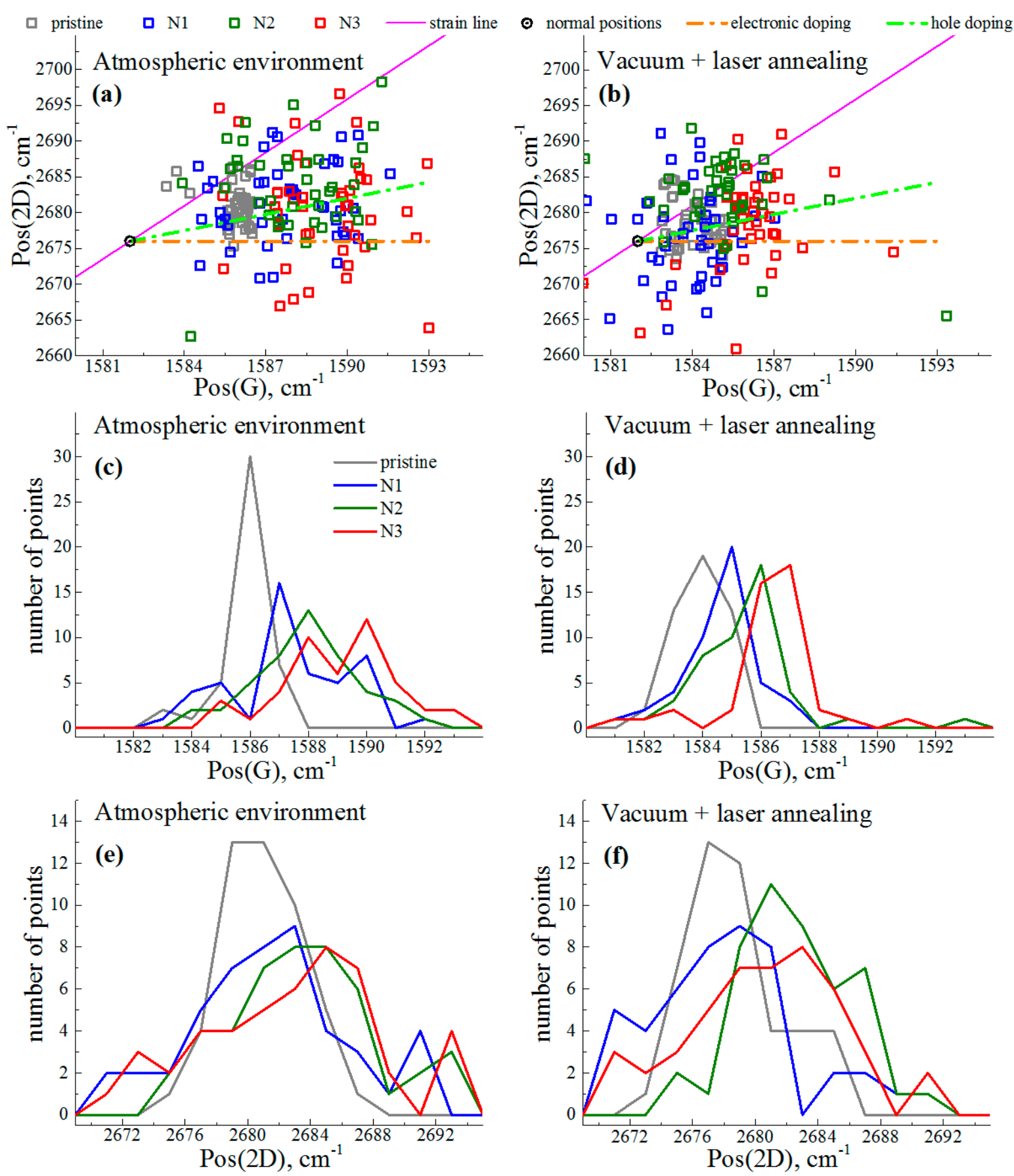

Figure 3. Diagrams of 2D and G Raman peak positions for pristine and nitrogen-doped graphene (a) under atmospheric environment, and (b) after laser annealing in vacuum (the point scatter is seen to reduce after the annealing due to adsorbate removal); the number of points corresponding to each peak position value (c) and (e) under atmospheric environment, and (d) and (f) after laser annealing in vacuum.

Table 2. Carrier density due to nitrogen doping calculated after XPS measurements $p_{\text {calc }}$, as well as the average carrier density change due to desorption in vacuum after laser annealing $\Delta p_{\text {exp }}$, for the experimental graphene.

\begin{tabular}{lll}
\hline Sample & $p_{\text {calc }}, \times 10^{13} \mathrm{~cm}^{-2}$ & $\Delta p_{\text {exp }}, \times 10^{13} \mathrm{~cm}^{-2}$ \\
\hline Pristine & 0 & 0.46 \\
N1 & 1.89 & 0.33 \\
N2 & 3.03 & 0.13 \\
N3 & 3.14 & 0.12 \\
\hline
\end{tabular}

results from the incorporated nitrogen concentration point of view, they do not have a linear relationship with the treatment time in terms of the carbon vacancy introduction and, therefore, D peak intensity, as in [25]. This can be explained by the fact that Raman and XPS spectra are typically measured in different areas: while the former is local $\left(6 \times 6 \mu \mathrm{m}^{2}\right.$ in the present work), the latter is more integral, applied in this case to $200 \times 200 \mu \mathrm{m}^{2}$. At the same time, the effects of plasma etching depend on the defect type [27], and are most pronounced in the areas where more initial defects are already 
present, such as grain boundaries. After the nitrogen incorporation reaches saturation with respect to time, the subsequent plasma treatment primarily leads to disordering of the already strongly defective regions, which is thermodynamically favorable. Simultaneously, at this stage, it has a smaller effect (related mostly to nitrogen removal) on more structured areas, which can be studied by Raman spectroscopy. Due to the scope of this work, the sample labelling thus follows the resultant nitrogen concentration and Raman data, rather than the treatment time.

Three common bonding configurations are normally obtained when incorporating nitrogen into the graphene lattice: pyridinic $\mathrm{N}$ ( $\mathrm{sp}^{2}$-hybridized), pyrrolic $\mathrm{N}$ ( $\mathrm{sp}^{3}$-hybridized) and graphitic $\mathrm{N}$ ( $\mathrm{sp}^{2}$-hybridized) [28]. According to the photoemission data presented in figure $2, \mathrm{~N}_{2}$ plasma treatment of graphene mainly leads to pyridinic and graphitic nitrogen incorporation. Pyridinic nitrogen is known to introduce about 0.45 holes per nitrogen atom, while graphitic nitrogen leads to electronic doping of $\sim 0.54$ electrons/atom [29]. The nature of $p$-type doping by pyridinic nitrogen is not due to the presence of the $\mathrm{N}$ atom itself, but is due to a carbon vacancy, which results in a lack of electrons in the $\pi$-system [24]. The amount of pyridinic nitrogen in the plasma-treated samples is substantially higher than that of graphitic nitrogen as measured by XPS (table 1), which obviously implies an overall $p$-type doping effect, in agreement with the Raman data presented in figure 1 . It should be noted that graphene transfer to a $\mathrm{SiO}_{2} /$ Si substrate can lead to an additional doping effect due to the interfacial charge redistribution [30], charge transfer from $\mathrm{H}_{2} \mathrm{O} / \mathrm{O}_{2}$ molecules [31] or an increase in external scattering sites due to the polymer residue [22].

Considering that the adsorbates present on the sample surface under atmospheric conditions introduce additional $p$-type doping of graphene [3-8], laser annealing with $10 \mathrm{~mW}$ power was performed for all samples in vacuum $\left(\sim 10^{-5} \mathrm{mbar}\right)$ for the adsorbate removal (see the experimental section for full details of the measurement/annealing/measurement sequence). It should be noted that for a sensitive material such as graphene, the vacuum condition itself is sufficient to observe noticeable changes in the Raman spectra [8]; laser annealing can be expected to enhance its effectiveness in relation to adsorbate removal through additional energy transfer. Moreover, the calculations given in [8] showed that the effects of graphene and substrate lattice compressibility on Raman spectra are negligible at pressures below atmospheric pressure.

Figures 3(a) and (b) present 2D-G diagrams for the experimental samples before and after laser annealing in vacuum. As was shown in [13,14], such diagrams are useful for visual doping descriptions in graphene due to nonlinear dependence of Raman peak positions on charge carrier density [12]. The latter allows us to highlight separate directions for $n$ - and $p$-type doping increases, as well as to distinguish doping and strain effects. As the vacuum condition and laser annealing lead to adsorbate removal from the sample surface, the point clouds experience a pronounced shift along the hole doping line seen in figures 3(a) and (b). Along with the change in the $I_{2 \mathrm{D}} / I_{\mathrm{G}}$ intensity ratio observed during the Raman experiments, this allows us to exclude the

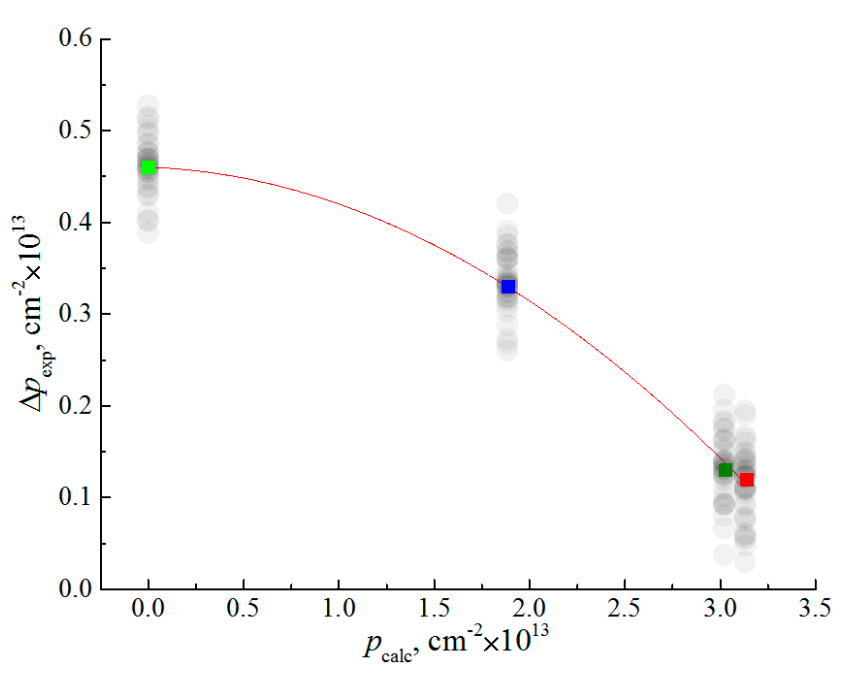

Figure 4. Dependence of the average carrier density change due to desorption in vacuum after laser annealing $\Delta p_{\text {exp }}$ on the carrier density due to nitrogen doping $p_{\text {calc }}$ calculated after XPS measurements. The solid line shows the general trend of the dependency and is given here for clarity of the data presented. The semi-transparent circles represent individual values for single Raman spectra obtained during the scanning.

strain factor from the considerations. However, while visualizing the hole density decrease, the 2D-G diagrams show the strong overall point scatter, especially for the atmospheric environment, where the points are strongly affected by a probabilistic adsorption process. Therefore, we also give the number of points corresponding to each peak position in figures 3(c)-(f), presenting individual peak shifts statistically and more clearly (note that the 2D peak experiences a weaker shift as the carrier density changes [12]). Apart from the shift, the effect of desorption is also evident in position distribution narrowing after the annealing, most noticeably for the G peak. As the adsorbates get removed, the effect of incorporated nitrogen can be distinguished: the initial doping of graphene is manifested in ascending arrangement of the point clouds along the hole doping line observed in figures 3(b), (d) and (f), corresponding to an increasing percentage of pyridinic nitrogen.

Since the electrons introduced in graphene by graphitic nitrogen partially compensate the $p$-doping effect of the pyridine nitrogen [29], it would be reasonable to calculate the resultant hole density in doped graphene based on XPS data $p_{\text {calc }}$; its values for the experimental graphene are presented in the second column of table 2. At the same time, it is possible to obtain a charge carrier density decrease in graphene during the desorption due to laser annealing in vacuum $\Delta p_{\text {exp }}$ from a combination of characteristic Raman spectra parameters (primarily the $\mathrm{G}$ and 2D peak positions) [12], which is presented in the third column of table 2, averaged over the scanned areas. The $I_{2 \mathrm{D}} / I_{\mathrm{G}}$ intensity ratios have generally increased by $0.03-0.09$ after laser annealing, indicating the charge carrier density decrease as well [12]; however, they were not informative for charge carrier density estimation since their values were also strongly affected by the destruction of the graphene $\mathrm{sp}^{2}$ lattice during nitrogen incorporation [11], as can be seen in figure 1 . 

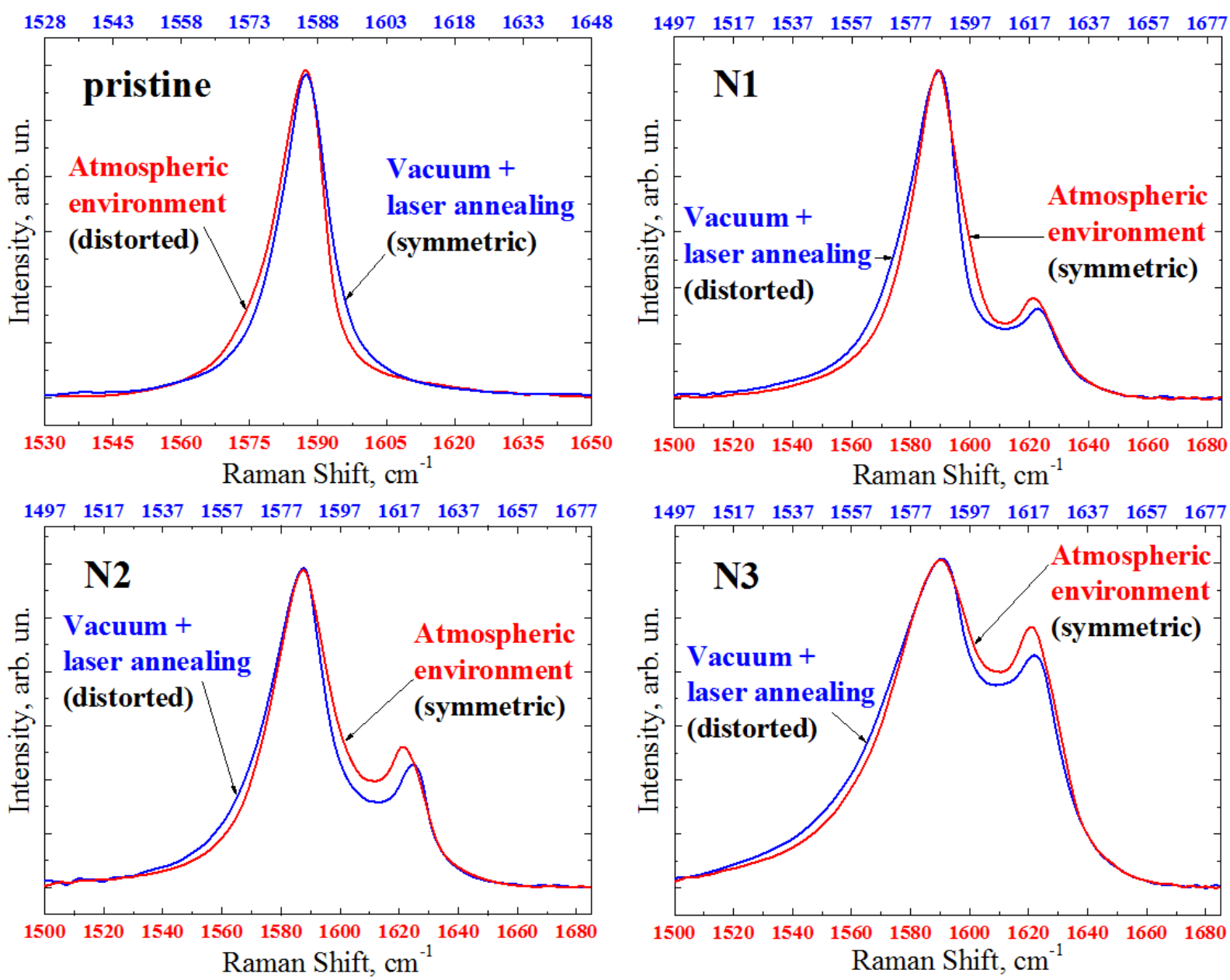

Figure 5. Typical Raman G peak profiles for pristine and nitrogen-doped graphene N1-N3 under an atmospheric environment and after laser annealing in vacuum.

It can be seen from table 2 that the $\Delta p_{\exp }$ values gradually decrease, which suggests that the adsorption softens with increasing initial $p$-type doping as the Fermi level of graphene is already below the Dirac point, and the pyridic nitrogen sites, which dominate over the graphitic ones in the studied samples, introduce charge carriers with the same signs as basic atmospheric acceptor $\mathrm{H}_{2} \mathrm{O}$ and $\mathrm{O}_{2}$ groups. The fact that doping with the same charge carrier sign as the adsorbate makes adsorption doping less energetically favorable is in conceptual agreement with other works on the subject, such as [32, 33]. Comparing this observation with the results presented in $[8,32]$ allows us to extend this assessment of the $n$-type doping, which contrariwise increases the adsorptivity, introducing impurity energy levels suitable for the electron exchange [32].

Figure 4 shows the dependence of the charge carrier density change after laser annealing on $p_{\text {calc }}$ obtained from XPS data, with the scatter of values on the scans (semi-transparent points were calculated for the peak positions at each scan point).

It is seen from figure 4 that the intersection of the dependency with a vertical axis allows us to determine the required amount of doping $p_{\text {noad }}$, which will expectedly lead to the absence of adsorption doping by introducing charge carriers with the same sign. The estimation gives $p_{\text {noad }}=(3.87 \pm$
$0.31) \times 10^{13} \mathrm{~cm}^{-2}$, which corresponds to $2.3 \%$ of pyridinelike nitrogen $\mathrm{N}_{\mathrm{P}}$.

In addition, the dependency intersection with a horizontal axis gives a required electronic doping $\mathrm{n}_{\text {equil }}$, which under an atmospheric environment results in an equilibrium state with a zero carrier density due to full compensation of adsorption doping. It can be estimated as $n_{\text {equil }}=(0.46 \pm 0.12)$ $\times 10^{13} \mathrm{~cm}^{-2}$, corresponding to $\sim 0.2 \%$ of graphitic nitrogen $\mathrm{N}_{\mathrm{C}}$. Graphene $n$-doped to smaller electronic density values is expected to change the charge carrier sign when exposed to the atmospheric environment. It should be noted that both $p_{\text {noad }}$ and $n_{\text {equil }}$ will be, in principle, substrate-dependent [8, $34,35]$ due to charge transfer at the interface [30], electronphonon coupling screening $[36,37]$ and other substraterelated effects.

Both doped graphene and graphene with adsorbates present on its surface tend towards an increase in the spatial charge carrier inhomogeneity, which is known to distort the $\mathrm{G}$ peak profile $[8,38]$. Figure 5 presents such profiles plotted for each sample, before and after laser annealing in vacuum; the peaks are shifted to overlap for better clarity, the lower scale relates to the atmospheric environment and the upper one to the annealed samples in vacuum, as indicated by the colors and arrows. 
It is seen from figure 5 that for pristine graphene, the $G$ peak is distorted under atmospheric conditions, while annealing in vacuum leads to its symmetrization. At the same time, the opposite is observed for doped graphene: a more symmetric $\mathrm{G}$ peak is observed here in the case of air-exposed samples. This leads to a very interesting conclusion. The presence of adsorption $p$-type doping leads to spatially inhomogeneous hole introduction, which distorts the $\mathrm{G}$ peak of pristine graphene as the adsorbates get attached to its surface. In the initially $p$-doped graphene, the adsorption process turns out to be spatially selective to balance the local charge carrier deficiencies arising from unevenly distributed nitrogen sites, thus reducing the inhomogeneity and at the same time introducing additional holes, increasing the overall charge carrier density. This logical but not obvious observation correlates with the results for $n$-doped graphene, in which selective adsorption $p$-type doping compensates both charge carrier inhomogeneity and density through the electron-hole recombinations [8].

In general, 2D materials are extremely sensitive, even to small external influences. This study additionally focuses attention on the fact that such a trivial and commonly neglected factor as an atmospheric environment notably affects the properties of graphene, changing its carrier density by values to the order of $10^{12} \mathrm{~cm}^{-2}$. Thus, precise engineering of nanoelectronic devices requires taking the described effects into account if their functional elements can be air-exposed.

\section{Conclusion}

In this work, we present Raman and photoemission spectroscopy studies of the adsorption properties of pristine graphene and graphene, $p$-doped with nitrogen. We found that laser annealing with $10 \mathrm{~mW}$ of power in vacuum $\left(\sim 10^{-5}\right.$ mbar $)$ led to a pronounced decrease in hole density due to acceptor adsorbate removal from the sample surface by $0.46,0.33,0.13$ and $0.12 \times 10^{13} \mathrm{~cm}^{-2}$ for pristine graphene and graphene with increasing doping, respectively. The shift inversely correlated with a degree of initial nitrogen doping, indicating that less $p$-type adsorption doping takes place for graphene with a higher density of charge carriers of the same sign. The amount of initial $p$-type doping required for the absence of atmospheric adsorption doping was estimated as $p_{\text {noad }}=(3.87 \pm 0.31) \times 10^{13} \mathrm{~cm}^{-2}$, corresponding to $\sim 2.3 \%$ of pyridine-like nitrogen. Furthermore, the electronic doping required to fully compensate adsorption hole doping under the atmospheric environment was $n_{\text {equil }}=(0.46 \pm 0.12) \times$ $10^{13} \mathrm{~cm}^{-2}$, corresponding to $\sim 0.2 \%$ of graphitic nitrogen. Finally, we found that adsorption leads to a distortion of the Raman $\mathrm{G}$ peak profile corresponding to an increase in spatial charge carrier inhomogeneity in pristine graphene, while for nitrogen-doped graphene, the opposite occurs: the adsorption turns out to be spatially selective to reduce the inhomogeneity. This study is useful for graphene applications which require certain adsorption properties, as well as for taking these properties into account when designing graphene-based nanoelectronic devices.

\section{Acknowledgments}

This work was supported by the Belarusian State Program for Research 'Photonics, opto- and microelectronics', the Russian Foundation for Basic Research (RFBR No. 19-2903050 and 18-29-19120) and was partially supported by the Basic Science Research Program (2016R1A6A1A03012877; 2017R1D1A1B03035102; 2017R1D1A1B03032759) through the National Research Foundation of Korea (NRF).

\section{ORCID iDs}

Mikhail S Tivanov (D) https://orcid.org/0000-0002-4243-0813 Gennady N Panin (1) https://orcid.org/0000-0003-3658-6958

\section{References}

[1] Geim A K and Novoselov K S 2007 Nat. Mater. 6 183-91

[2] Shao Y, Wang J, Wu H, Liu J, Aksay I A and Lin Y 2010 Electroanalytical 22 1027-36

[3] Ryu S, Liu L, Berciaud S, Yu Y-J, Liu H, Kim P, Flynn G W and Brus L E 2010 Nano Lett. 10 4944-51

[4] Kong L, Enders A, Rahman T S and Dowben P A 2014 J. Phys.: Condens. Matter 26443001

[5] Yang Y, Brenner K and Murali R 2012 Carbon 50 1727-33

[6] Pinto H and Markevich A 2014 Beilstein J. Nanotechnol. 5 1842-8

[7] Kalkan S B, Aydin H, Özkendir D and Çelebi C 2018 Appl. Phys. Lett. 112013103

[8] Kolesov E A, Tivanov M S, Korolik O V, Kapitanova O O, Fu X, Cho H D, Kang T W and Panin G N 2018 Beilstein J. Nanotechnol. 9 704-10

[9] Yazdi G R, Akhtar F, Ivanov I G, Schmidt S, Shtepliuk I, Zakharov A, Iakimov T and Yakimova R 2019 Appl. Surf. Sci. 486 239-48

[10] Dai J and Yuan J 2010 Phys. Rev. B 81165414

[11] Ferrari A C and Basko D M 2013 Nat. Nanotechnol. 8235

[12] Beams R, Cançado L G and Novotn L 2015 J. Phys.: Condens. Matter 27083002

[13] Bartolomé J, Álvarez-Fraga L, Aguilar-Pujol M X, Cortijo S, Cremades A, Prieto C and de Andrés A 2019 2D Mater. 6015023

[14] Lee J E, Ahn G, Shim J, Lee Y S and Ryu S 2012 Nat. Commun. 31024

[15] Susi T, Pichler T and Ayala P 2015 Beilstein J. Nanotechnol. 6 177-92

[16] Günes F, Han G H, Kim K K, Kim E S, Chae S J, Park M H, Jeong H-K, Lim S C and Lee Y H 2009 Nano 483

[17] Liang X et al 2011 ACS Nano 59144

[18] Gao L, Ni G-X, Liu Y, Liu B, Castro Neto A H and Loh K P 2014 Nature $\mathbf{5 0 5} 190$

[19] Her M, Beams R and Novotny L 2013 Phys. Lett. A 3771455

[20] Grüneis A, Kummer K and Vyalikh D V 2009 New J. Phys. 11073050

[21] Kapitanova O O et al 2017 J. Phys. Chem. C 121 27915-22

[22] Pirkle A et al 2011 Appl. Phys. Lett. 99122108

[23] Usachov D et al 2011 Nano Lett. 11 5401-7

[24] Usachov D et al 2014 Nano Lett. 14 4982-8

[25] Rybin M, Pereaslavtsev A, Vasilieva T, Myasnikov V, Sokolov I, Pavlova A, Obraztsova E, Khomich A, Ralchenko V and Obraztsova E 2016 Carbon 96 196-202

[26] Lin Y-P, Ksari Y, Prakash J, Giovanelli L, Valmalette J-C and Themlin J-M 2014 Carbon 73 216-24 
[27] Dong Y, Gahl M T, Zhang C and Lin J 2017 Nanotechnology 28505602

[28] Wang H, Maiyalagan T and Wang X 2012 ACS Catal. 2 781-94

[29] Schiros T et al 2012 Nano Lett. 12 4025-31

[30] Shi Y, Dong X, Chen P, Wang J and Li L-J 2009 Phys. Rev. B 79115402

[31] Levesque P L, Sabri S S, Aguirre C M, Guillemette J, Siaj M, Desjardins P, Szkopek T and Martel R 2011 Nano Lett. 11 132-7

[32] Wang W, Zhang Y, Shen C and Chai Y 2016 AIP Adv. 6025317

[33] Yang S, Lei G, Xu H, Xu B, Li H, Lan Z, Wang Z and Hu G 2019 Appl. Surf. Sci. 480 205-11
[34] Smerieri M, Celasco E, Carraro G, Lusuan A, Pal J, Bracco G, Rocca M, Savio L and Vattuone L 2015 ChemCatChem 7 2328-31

[35] Celasco E, Carraro G, Lusuan A, Smerieri M, Pal J, Rocca M, Savio L and Vattuone L 2016 Phys. Chem. Chem. Phys. 18 18692-6

[36] Siegel D A, Hwang C, Fedorov A V and Lanzara A 2012 New J. Phys. 14095006

[37] Attaccalite C, Wirtz L, Lazzeri M, Mauri F and Rubio A 2010 Nano Lett. 101172

[38] Casiraghia C, Pisana S, Novoselov K S, Geim A K and Ferrari A C 2007 Appl. Phys. Lett. 91233108 